\title{
Quotients of non-classical flag domains are not algebraic
}

\author{
Phillip Griffiths, Colleen Robles and Domingo Toledo
}

\begin{abstract}
A flag domain $D=G / V$ for $G$ a simple real non-compact group $G$ with compact Cartan subgroup is non-classical if it does not fiber holomorphically or anti-holomorphically over a Hermitian symmetric space. We prove that for $\Gamma$ an infinite, finitely generated discrete subgroup of $G$, the analytic space $\Gamma \backslash D$ does not have an algebraic structure. We also give another proof of the theorem of Huckleberry that any two points in a non-classical domain $D$ can be joined by a finite chain of compact subvarieties of $D$.
\end{abstract}

\section{Introduction}

The purpose of this paper is to prove that if $D$ is a non-classical flag domain and $\Gamma$ is an infinite, finitely generated, discrete group of automorphisms of $D$, then the quotient space $\Gamma \backslash D$ is not an algebraic variety. We also discuss some geometry of these domains.

By flag domain we mean a homogeneous complex manifold $D=G / V$ for a non-compact, connected, linear, real semisimple Lie group $G$ with a compact Cartan subgroup $T$, where the isotropy group $V$ is the centralizer of a subtorus of $T$. Note that our terminology includes a wider class than the manifolds $G / T$. It will be convenient to assume that $G$ is simple and adjoint, and leave the formulation and proofs of the corresponding results in the general semi-simple case to the reader.

There is a unique maximal compact subgroup $K$ of $G$ containing $V$ and we have inclusions $T \subset V \subset K \subset G$. We have a fibration $p: G / V \rightarrow G / K$ of $D$ to the symmetric space $G / K$ for $G$ with fiber $K / V$. We say that the domain $D=G / V$ is classical if $G / K$ is Hermitian symmetric and $p$ is holomorphic or anti-holomorphic. Otherwise $D$ is said to be non-classical.

Theorem 1.1. Let $D$ be a non-classical domain, with $G$ simple and adjoint, and let $\Gamma \subset G$ be an infinite, finitely generated discrete subgroup. Then the normal complex analytic space $X=\Gamma \backslash D$ does not have an algebraic structure.

We say that a complex manifold (or an analytic space) $X$ has an algebraic structure if $X$ is biholomorphic to the analytic space $W^{a n}$ associated to an abstract algebraic variety $W$ over $\mathbb{C}$

Received 7 March 2013, accepted in final form 1 July 2013.

2010 Mathematics Subject Classification 53C30, 58A14

Keywords: Hodge theory, flag domain.

This journal is (C) Foundation Compositio Mathematica 2014. This article is distributed with Open Access under the terms of the Creative Commons Attribution Non-Commercial License, which permits non-commercial reuse, distribution, and reproduction in any medium, provided that the original work is properly cited. For commercial re-use, please contact the Foundation Compositio Mathematica.

Robles is partially supported by NSF DMS-1006353, Toledo is partially supported by Simons Foundation grant 208853. 


\section{Griffiths, Robles And Toledo}

(or a scheme $W$ of finite type over $\mathbb{C}$ ). For example, a quasiprojective variety has an algebraic structure in this sense.

The motivation for Theorem 1.1 comes from the study of variations of Hodge structure and period mappings for families of algebraic varieties $\mathcal{V} \rightarrow S$. In this situation there is a flag domain $D$, the period domain, and a holomorphic map $S \rightarrow X=\Gamma \backslash D$, the period map, where $\Gamma \subset G$ is the monodromy group. If the Hodge structure has weight one, then $D$ is a Hermitian symmetric domain, but if it has higher weight, then $D$ is typically a non-classical domain.

One of the ways it was realized that Hodge structures of higher weight are fundamentally different from those of weight one is that there are no automorphic forms on their period domains, meaning that $H^{0}\left(X, \omega_{X}^{\otimes k}\right)=0$ for all $k>0$. This led to the general suspicion, never proved, that $X$ is not an algebraic variety. Theorem 1.1 confirms that this is indeed the case.

It is worth noting that $X$ contains algebraic subvarieties. Besides the images of the $Z_{u} \subset D$ defined below, it contains, e.g., images of period mappings $S \rightarrow X$, and quotients of equivariantly embedded Hermitian symmetric domains $D^{\prime} \subset D$. The geometry of such non-algebraic varieties containing many positive-dimensional algebraic subvarieties is a subject of current interest.

Note that Theorem 1.1 is known in the case that $\Gamma$ is co-compact [CT12]. The point of this paper is to treat the non-co-compact case, which is the significant one for Hodge theory, since the images of monodromy representations are typically not co-compact.

The proof of Theorem 1.1 proceeds as follows. First, the fibers of $p: D \rightarrow G / K$ are complex subvarieties of $D$, biholomorphic to the homogeneous projective variety $K / V$. It is well known that $D$ is non-classical if and only if it has compact subvarieties that are not contained in the fibers of $p$. In this case there is a family $Z_{u} \subset D$ of complex subvarieties, which are deformations of the fibers of $p$, parametrized by a Stein manifold $\mathcal{U}$ see, for example, [FHW06, Gr12, WW77].

Then we use the following theorem, which is a special case of Huckleberry's Proposition 3.1 [Hu13]:

Theorem 1.2. Let $D$ be a non-classical domain, with $G$ simple, and let $x, y \in D$. Then there exists a finite sequence $u_{1}, \ldots, u_{k} \in \mathcal{U}$ so that $x \in Z_{u_{1}}, y \in Z_{u_{k}}$ and $Z_{u_{i}} \cap Z_{u_{i+1}} \neq \emptyset$ for $i=1, \ldots, k-1$.

Reducing to torsion free $\Gamma$, since the subvarieties $Z_{u}$ are rational, this implies that the complex manifold $\Gamma \backslash D$ is rationally chain connected. Compact rationally connected algebraic manifolds have finite fundamental group, see $\S 2$ of [Cam91]. It was pointed out to us by Kollár that, as an easy consequence of the properties of the Shafarevich map [Ko95], finiteness also holds for noncompact algebraic varieties. Since $\pi_{1}(\Gamma \backslash D)$ is the infinite group $\Gamma$, this precludes the existence of an algebraic structure. This proves Theorem 1.1. Kollár also points out that the conclusion of Theorem 1.1 can be strengthened to say that $\Gamma \backslash D$ is not a Zariski open set in a complex space of class $\mathcal{C}$.

Huckleberry's proof of Theorem 1.2 is a very elegant argument within the framework of his paper. We have chosen to include our own argument, which presents a complementary perspective on the geometry of the maximal compact subvarieties in a flag domain. It is based on the bracket generating properties of various sub-bundles of the tangent bundle of the incidence variety (10), namely Lemma 2.6 and Proposition 4.2. We feel that this is interesting and useful information that will be needed in future work, e.g., in the study of how the $Z_{u}$ 's approach the boundary of $D$. 


\section{NON-CLASSICAL DOMAINS}

\section{Flag Domains}

\subsection{Lie theory preliminaries}

In this section we set notation and review some Lie theory associated to the flag domain $G / V$. Let $\mathfrak{t} \subset \mathfrak{v} \subset \mathfrak{k} \subset \mathfrak{g}$ denote the Lie algebras of $T \subset V \subset K \subset G$. Let $\mathfrak{g}=\mathfrak{k} \oplus \mathfrak{q}$ be the Cartan decomposition. In particular, the Killing form $B$ is negative definite on $\mathfrak{k}$ and positive definite on $\mathfrak{q}$, and

$$
[\mathfrak{k}, \mathfrak{q}] \subset \mathfrak{q} \text { and }[\mathfrak{q}, \mathfrak{q}] \subset \mathfrak{k} \text {. }
$$

Given a subspace $\mathfrak{a} \subset \mathfrak{g}$, let $\mathfrak{a}_{\mathbb{C}}$ denote the complexification. Then $\mathfrak{h}=\mathfrak{t}_{\mathbb{C}}$ is a Cartan subalgebra of $\mathfrak{g}_{\mathbb{C}}$. Let $\Delta=\Delta\left(\mathfrak{g}_{\mathbb{C}}, \mathfrak{h}\right)$ denote the roots of $\mathfrak{g}_{\mathbb{C}}$. Given a root $\alpha \in \Delta$, let $\mathfrak{g}^{\alpha} \subset \mathfrak{g}_{\mathbb{C}}$ denote the associated root space. Given a subspace $\mathfrak{s} \subset \mathfrak{g}_{\mathbb{C}}$, define

$$
\Delta(\mathfrak{s})=\left\{\alpha \in \Delta \mid \mathfrak{g}^{\alpha} \subset \mathfrak{s}\right\} .
$$

If $[\mathfrak{h}, \mathfrak{s}] \subset \mathfrak{s}$, then we have

$$
\mathfrak{s}=(\mathfrak{s} \cap \mathfrak{h}) \oplus \bigoplus_{\alpha \in \Delta(\mathfrak{s})} \mathfrak{g}^{\alpha}
$$

In particular, it follows that

$$
\begin{aligned}
& \mathfrak{v}_{\mathbb{C}}=\mathfrak{h} \oplus \bigoplus_{\alpha \in \Delta\left(\mathfrak{v}_{\mathbb{C}}\right)} \mathfrak{g}^{\alpha}, \\
& \mathfrak{k}_{\mathbb{C}}=\mathfrak{h} \oplus \bigoplus_{\alpha \in \Delta\left(\mathfrak{k}_{\mathbb{C}}\right)} \mathfrak{g}^{\alpha}, \\
& \mathfrak{q}_{\mathbb{C}}=\bigoplus_{\alpha \in \Delta\left(\mathfrak{q}_{\mathbb{C}}\right)} \mathfrak{g}^{\alpha} .
\end{aligned}
$$

Fix a Borel subalgebra $\mathfrak{h} \subset \mathfrak{b} \subset \mathfrak{g}_{\mathbb{C}}$. As above, we have

$$
\mathfrak{b}=\mathfrak{h} \oplus \bigoplus_{\alpha \in \Delta(\mathfrak{b})} \mathfrak{g}^{\alpha}
$$

Moreover, note that

$$
\Delta^{+}=\Delta(\mathfrak{b})
$$

determines a set of positive roots. Let $\left\{\sigma_{1}, \ldots, \sigma_{r}\right\} \subset \Delta^{+}$denote the corresponding simple roots. Let $\left\{\mathrm{T}^{1}, \ldots, \mathrm{T}^{r}\right\}$ denote the basis of $\mathfrak{h}$ dual to the simple roots,

$$
\sigma_{i}\left(\mathrm{~T}^{j}\right)=\delta_{i}^{j} .
$$

Let $\Delta\left(\mathfrak{v}_{\mathbb{C}}\right)^{\perp}=\Delta \backslash \Delta(\mathfrak{v})$. Define

$$
\mathrm{T}=\sum_{\sigma_{i} \in \Delta\left(\mathfrak{q}_{\mathbb{C}}\right)} \mathrm{T}^{i}+\sum_{\sigma_{i} \in \Delta\left(\mathfrak{k}_{\mathbb{C}}\right) \backslash \Delta\left(\mathfrak{v}_{\mathbb{C}}\right)} 2 \mathrm{~T}^{i} .
$$

Since $\mathrm{T} \in \mathfrak{h}$ is semisimple, the Lie algebra $\mathfrak{g}_{\mathbb{C}}$ decomposes as a direct sum of $\mathrm{T}$-eigenspaces

$$
\mathfrak{g}_{\mathbb{C}}=\mathfrak{g}_{k} \oplus \mathfrak{g}_{k-1} \oplus \cdots \oplus \mathfrak{g}_{1-k} \oplus \mathfrak{g}_{-k},
$$

where

$$
\mathfrak{g}_{\ell}=\left\{\xi \in \mathfrak{g}_{\mathbb{C}} \mid[\mathrm{T}, \xi]=\ell \xi\right\} .
$$

Note that, as the roots are integral linear combinations of the simple roots, (2) and (3) imply that the eigenvalues $\ell$ are integers. 


\section{Griffiths, Robles And Toledo}

Remark. Since the roots are pure imaginary on the compact Cartan subalgebra $t$, it follows that $\mathrm{T} \in \mathbf{i t}$, where $\mathbf{i}=\sqrt{-1}$. Therefore,

$$
\overline{\mathfrak{g}_{k}}=\mathfrak{g}_{-k} .
$$

It follows from (4) and (5) that $\mathfrak{g}^{k,-k}=\mathfrak{g}_{k}$ defines a real, weight zero Hodge structure on $\mathfrak{g}$. Moreover, the fact that $\mathfrak{g}=\mathfrak{k} \oplus \mathfrak{q}$ is a Cartan decomposition implies that this Hodge structure is polarized by the Killing form $B$.

By the Jacobi identity, we have

$$
\left[\mathfrak{g}_{\ell}, \mathfrak{g}_{m}\right] \subset \mathfrak{g}_{\ell+m} .
$$

We call $\mathrm{T}$ a grading element, and the eigenspace decomposition (4) the T-graded decomposition of $\mathfrak{g}_{\mathbb{C}}$. Observe that

$$
\begin{aligned}
\mathfrak{h} \subset \mathfrak{v}_{\mathbb{C}} & =\mathfrak{g}_{0}, \\
\mathfrak{k}_{\mathbb{C}} & =\mathfrak{g}_{\text {even }}=\oplus \mathfrak{g}_{2 \ell}, \\
\mathfrak{q}_{\mathbb{C}} & =\mathfrak{g}_{\text {odd }}=\oplus \mathfrak{g}_{2 \ell+1} .
\end{aligned}
$$

It will be convenient to write

$$
\mathfrak{g}_{+}=\bigoplus_{\ell>0} \mathfrak{g}_{\ell} \quad \text { and } \quad \mathfrak{g}_{-}=\bigoplus_{\ell>0} \mathfrak{g}_{-\ell} .
$$

Likewise, $\mathfrak{k}_{\mathbb{C}}=\mathfrak{k}_{+} \oplus \mathfrak{k}_{0} \oplus \mathfrak{k}_{-}$and $\mathfrak{q}_{\mathbb{C}}=\mathfrak{q}_{+} \oplus \mathfrak{q}_{-}$, with $\mathfrak{k}_{0}=\mathfrak{g}_{0}$,

$$
\mathfrak{k}_{ \pm}=\mathfrak{k}_{\mathbb{C}} \cap \mathfrak{g}_{ \pm} \quad \text { and } \quad \mathfrak{q}_{ \pm}=\mathfrak{q}_{\mathbb{C}} \cap \mathfrak{g}_{ \pm}
$$

Let

$$
\mathfrak{m}_{\ell}=\mathfrak{g} \cap\left(\mathfrak{g}_{\ell} \oplus \mathfrak{g}_{-\ell}\right)
$$

then one has

$$
\mathfrak{g}=\mathfrak{v} \oplus \mathfrak{m}_{1} \oplus \cdots \oplus \mathfrak{m}_{k}
$$

The real tangent bundle $T D$ is the homogeneous vector bundle

$$
T D=G \times_{V}(\mathfrak{g} / \mathfrak{v})=G \times_{V} \mathfrak{m}_{+},
$$

where $\mathfrak{m}_{+}=\mathfrak{m}_{1} \oplus \cdots \oplus \mathfrak{m}_{k}$. A homogeneous complex structure on $D$ is given by specifying $T_{\mathbb{C}} D=T_{1,0} D \oplus T_{0,1} D$ with

$$
T_{1,0} D=G \times_{V} \mathfrak{g}_{-} \text {and } T_{0,1} D=G \times_{V} \mathfrak{g}_{+} .
$$

\subsection{The compact dual}

Equation (6) implies that

$$
\mathfrak{p}=\mathfrak{g}_{\geqslant 0}=\mathfrak{v}_{\mathbb{C}}+\mathfrak{b}
$$

is a Lie subalgebra of $\mathfrak{g}_{\mathbb{C}}$. Moreover, $\mathfrak{b} \subset \mathfrak{p}$ implies that $\mathfrak{p}$ is a parabolic Lie algebra. Let $G_{\mathbb{C}}$ be the complexification of $G$, and let $P \subset G_{\mathbb{C}}$ be the parabolic subgroup with Lie algebra $\mathfrak{p}$. Then the flag domain $D=G / V$ may be identified with the open $G$-orbit of $P / P$ in the compact dual $\check{D}=G_{\mathbb{C}} / P$.

A $G_{\mathbb{C}}$-homogeneous complex structure on $\check{D}$ is given by

$$
T_{1,0} \check{D}=G_{\mathbb{C}} \times_{P}(\mathfrak{g} / \mathfrak{p}) \text { and } T_{0,1} \check{D}=G_{\mathbb{C}} \times_{P}(\overline{\mathfrak{g} / \mathfrak{p}}) .
$$

From (6) and (8), it is immediate that $\mathfrak{g} / \mathfrak{p} \simeq \mathfrak{g}_{+}$is a $\mathfrak{p}$-module identification; thus, $T_{0,1} \check{D}=$ $G_{\mathbb{C}} \times{ }_{P} \mathfrak{g}_{+}$. Likewise, the vector space identification $\mathfrak{g} / \mathfrak{p} \simeq \mathfrak{g}_{-}$, and the fact that $\mathfrak{g} / \mathfrak{p}$ is a $\mathfrak{p}$-module, 


\section{NON-CLASSICAL DOMAINS}

allows us to view $\mathfrak{g}_{-}$as a $\mathfrak{p}$-module, and write $T_{1,0}=G_{\mathbb{C}} \times_{P} \mathfrak{g}_{-}$. In particular, the restriction of the $G_{\mathbb{C}}$-homogeneous complex structure $(9)$ on $\check{D}$ to $D$ agrees with the $G_{\mathbb{R}}$-homogeneous complex structure (7) on $D$.

Let

$$
\mathcal{T} D=T_{1,0} D \quad \text { and } \quad \mathcal{T} \check{D}=T_{1,0} \check{D}
$$

denote the holomorphic tangent bundles.

\subsection{Compact subvarieties}

Consider the natural fibration $p: G / V \rightarrow G / K$. The fiber

$$
Z=p^{-1}(K / K)=K / V .
$$

is a compact, complex submanifold of $D$. If $K_{\mathbb{C}}$ is the complexification of $K$, then

$$
Z=K_{\mathbb{C}} /\left(K_{\mathbb{C}} \cap P\right) .
$$

Lemma 2.1. Suppose $G$ is simple, and let $U \subset G_{\mathbb{C}}$ be a neighborhood of the identity with the property that $g Z \subset D$ for all $g \in G_{\mathbb{C}}$. Then $D$ is classical if and only if for every $g \in U, g Z$ is a fiber of $p$.

Proof. This is contained in Proposition 2.3.5 of [WW77], where the largest subgroup $L \subset G$ preserving $Z$ is determined.

Suppose $D$ is non-classical, and let $U$ be the maximal connected neighborhood of the identity in $G_{\mathbb{C}}$ with $g Z \subset D$ for all $g \in U$. Observe that $U$ is invariant under the right action of $K_{\mathbb{C}}$.

Definition 2.2. Assume $D$ to be non-classical. Define

$$
\mathcal{U}=U / K_{\mathbb{C}} \subset G_{\mathbb{C}} / K_{\mathbb{C}}
$$

this is the parameter space for the deformations $g Z \subset D$ of $Z$. Given $u=g K_{\mathbb{C}} \in \mathcal{U}$, let

$$
Z_{u}=g Z \subset D
$$

denote the corresponding variety. The incidence variety is

$$
\mathcal{I}=\left\{(x, u) \in D \times \mathcal{U} \mid x \in Z_{u}\right\} .
$$

The natural projections $\mathcal{I} \rightarrow D$ and $\mathcal{I} \rightarrow \mathcal{U}$ yield a diagram:

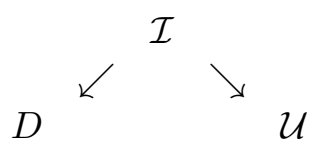

The proof of Theorem 1.2 utilizes three sub-bundles of the the holomorphic tangent bundle $\mathcal{T} \mathcal{I}$. First, observe that the holomorphic tangent bundle $\mathcal{T} \mathcal{I} \subset \mathcal{T}(D \times \mathcal{U})$ is the following subbundle

$$
\mathcal{T}_{(x, u)} \mathcal{I}=\left\{(\dot{x}, \dot{u}) \in \mathcal{T}_{x} D \oplus \mathcal{T}_{u} \mathcal{U} \mid \dot{u}(x) \equiv \dot{x} \bmod T_{x} Z_{u}\right\}
$$

Above, the tangent vector $\dot{u} \in \mathcal{T}_{u} \mathcal{U}$ is viewed as a holomorphic section of the normal bundle $N_{Z_{u} / D}=\mathcal{T} D / \mathcal{T} Z_{u}$; thus, the the normal field $\dot{u}$ can be evaluated at any $x \in Z_{u}$ to give a vector in $\mathcal{T}_{x} D / \mathcal{T}_{x} Z_{u}$. Geometrically, $\mathcal{T}_{(x, u)} \mathcal{I}$ is the set of tangent vectors $(\dot{x}(0), \dot{u}(0))$ given by curves $(x(t), u(t)) \in D \times \mathcal{U}$ with $x(t) \in Z_{u(t)}$ and $(x(0), u(0))=(x, u)$. 


\section{Griffiths, Robles And Toledo}

Definition 2.3. Define sub-bundles $S, E$ and $F$ of $T \mathcal{I}$ by:

$$
\begin{aligned}
S_{(x, u)} & =\left\{(\dot{x}, \dot{u}) \in T_{(x, u)} \mathcal{I} \mid \dot{x} \in T_{x} Z_{u}\right\} \\
& =\left\{(\dot{x}, \dot{u}) \in T_{(x, u)} \mathcal{I} \mid \dot{u}(x)=0\right\}, \\
E_{(x, u)} & =\left\{(\dot{x}, 0) \in T_{(x, u)} \mathcal{I}\right\}=\left\{(\dot{x}, 0) \in T_{x} D \oplus T_{u} \mathcal{U} \mid \dot{x} \in T_{x} Z_{u}\right\}, \\
F_{(x, u)} & =\left\{(0, \dot{u}) \in T_{(x, u)} \mathcal{I}\right\}=\left\{(0, \dot{u}) \in T_{x} D \oplus T_{u} \mathcal{U} \mid \dot{u}(x)=0\right\} .
\end{aligned}
$$

Note that the asserted equalities in the definitions follow from the defining equation (11) of $T \mathcal{I}$.

Remark 2.4. The geometric interpretation of these bundles is as follows. Let $(x(t), u(t))$ be a local holomorphic curve in $\mathcal{I}$. Then

- $(\dot{x}(t), \dot{u}(t)) \in S$ for all $t$ if and only if $\dot{x}(t) \in T_{x(t)} Z_{u(t)}$. In other words, $x$ stays in $Z_{u}$ to first order.

- $(\dot{x}(t), \dot{u}(t)) \in E$ for all $t$ if and only if $u(t)$ is constant $\equiv u$ and $x(t) \in Z_{u}$ for all $t$. In other words, $E$ is the bundle tangent to the fibers of $p_{\mathcal{U}}: \mathcal{I} \rightarrow \mathcal{U}$.

- $(\dot{x}(t), \dot{u}(t)) \in F$ for all $t$ if and only if $x(t)$ is constant $\equiv x$ and $x \in Z_{u(t)}$ for all $t$. In other words, $F$ is the bundle tangent to the fibers of the projection $p_{D}: \mathcal{I} \rightarrow D$.

Lemma 2.5. The sub-bundles $E$ and $F$ are integrable, and $S=E \oplus F$.

Lemma 2.6. The sub-bundle $S$ is bracket-generating.

Recall that a sub-bundle $S$ of the tangent bundle $T M$ of a manifold $M$ is called bracket generating if, for every $x \in M$, the evaluation map $\mathcal{L}_{x} \rightarrow T_{x} M$ is surjective, where $\mathcal{L}_{x}$ is the Lie algebra of germs of vector fields generated by the germs at $x$ of sections of $S$.

Lemmas 2.5 and 2.6 are proved using Lie algebra descriptions of the bundles above. First, observe that each of the spaces in (10) is an open set in a corresponding homogenous complex manifold. In fact, the diagram (10) embeds in the diagram

$$
\check{D}=G_{\mathbb{C}} / P{ }^{\swarrow{ }^{\check{\mathcal{I}}=G_{\mathbb{C}} /\left(K_{\mathbb{C}} \cap P\right)}} \searrow \check{\mathcal{U}}=G_{\mathbb{C}} / K_{\mathbb{C}}
$$

Moreover, the bundles $S, E, F$ of Definition (2.3) extend to homogeneous vector bundles $\check{S}, \check{E}, \check{F}$ over $\check{\mathcal{I}}$. Indeed,

$$
\mathcal{T} \check{\mathcal{I}}=G_{\mathbb{C}} \times_{K_{\mathbb{C}} \cap P}\left(\mathfrak{g}_{\mathbb{C}} / \mathfrak{k}_{\mathbb{C}} \cap \mathfrak{p}\right)=G_{\mathbb{C}} \times_{K_{\mathbb{C}} \cap P}\left(\mathfrak{g}_{\mathbb{C}} / \mathfrak{k}_{0} \oplus \mathfrak{k}_{+}\right) .
$$

Use the vector space identification $\mathfrak{g}_{\mathbb{C}} / \mathfrak{k}_{0} \oplus \mathfrak{k}_{+} \simeq \mathfrak{k}_{-} \oplus \mathfrak{q}=\mathfrak{k}_{-} \oplus \mathfrak{q}-\oplus \mathfrak{q}_{+}$to regard $\mathfrak{k}_{-} \oplus \mathfrak{q}$ as a $K_{\mathbb{C}} \cap P$-module. Then we mildly abuse notation by writing

$$
\mathcal{T} \check{\mathcal{I}}=G_{\mathbb{C}} \times_{K_{\mathbb{C}} \cap P}\left(\mathfrak{k}_{-} \oplus \mathfrak{q}_{-} \oplus \mathfrak{q}_{+}\right) .
$$

With respect to this identification, we have

$$
\begin{aligned}
\check{S} & =G_{\mathbb{C}} \times_{K_{\mathbb{C}} \cap P}\left(\mathfrak{k}_{-} \oplus \mathfrak{q}_{+}\right), \\
\check{E} & =G_{\mathbb{C}} \times_{K_{\mathbb{C}} \cap P} \mathfrak{k}_{-}, \\
\check{F} & =G_{\mathbb{C}} \times_{K_{\mathbb{C}} \cap P} \mathfrak{q}_{+} .
\end{aligned}
$$

The bundles $\mathcal{T} \mathcal{I}, S, E$ and $F$ are the restrictions of $\mathcal{T} \check{\mathcal{I}}, \check{S}, \check{E}$ and $\check{F}$ to $\mathcal{I} \subset \check{\mathcal{I}}$.

Proof of Lemma 2.5. It is immediate from (14) that $\check{S}=\check{E} \oplus \check{F}$; thus $S=E \oplus F$. Since $E, F$ are tangent to the fibers of the projections (10) they are clearly integrable. Alternatively, to see 


\section{NON-CLASSICAL DOMAINS}

that $\check{E}$ and $\check{F}$ are involutive, it suffices to show that

$$
\left[\mathfrak{k}_{-}, \mathfrak{k}_{-}\right] \subset \mathfrak{k}_{-} \bmod \mathfrak{k}_{\mathbb{C}} \cap \mathfrak{p}, \quad \text { and }\left[\mathfrak{q}_{+}, \mathfrak{q}_{+}\right] \subset \mathfrak{q}_{+} \quad \bmod \mathfrak{k}_{\mathbb{C}} \cap \mathfrak{p} .
$$

These two relations are straightforward consequences of the identities in $\S 2.1$.

The proof of Lemma 2.6 is more involved.

\section{Proof of Lemma 2.6}

By (13) and (14), the bundle $\check{S}$ is bracket-generating (equivalently, $S$ is bracket-generating) if and only if $\mathfrak{q}_{-}$is contained in the algebra generated by $\mathfrak{k}_{-} \oplus \mathfrak{q}_{+}$. Since $\mathfrak{g}_{-}=\mathfrak{k}_{-} \oplus \mathfrak{q}_{-}$, this is equivalent to

$$
\mathfrak{g}_{-} \text {is contained in the algebra generated by } \mathfrak{k}_{-} \oplus \mathfrak{q}_{+} \text {. }
$$

It will be helpful to define an auxiliary grading element

$$
\mathrm{T}^{\prime}=\sum_{\sigma_{i} \in \Delta\left(\mathfrak{q}_{\mathbb{C}}\right)} \mathrm{T}^{i}
$$

Let $\mathfrak{g}_{\mathbb{C}}=\mathfrak{g}_{a}^{\prime} \oplus \mathfrak{g}_{a-1}^{\prime} \oplus \cdots \oplus \mathfrak{g}_{1-a}^{\prime} \oplus \mathfrak{g}_{-a}^{\prime}$ be the $\mathrm{T}^{\prime}$-graded decomposition, cf. (4). By [CS09, Theorem 3.2.1(1)],

$$
\left.\mathfrak{g}_{+}^{\prime}\left(\text { resp., } \mathfrak{g}_{-}\right) \text {is generated by } \mathfrak{g}_{1}^{\prime} \text { (resp., } \mathfrak{g}_{-1}^{\prime}\right) .
$$

A similar argument implies that

$$
\left.\mathfrak{g}_{+}\left(\text {resp., } \mathfrak{g}_{-}\right) \text {is generated by } \mathfrak{g}_{1} \oplus \mathfrak{g}_{2} \text { (resp., } \mathfrak{g}_{-1} \oplus \mathfrak{g}_{-2}\right) \text {. }
$$

Therefore, by $\mathfrak{g}_{-2} \subset \mathfrak{k}_{-}$and (15), $\check{S}$ is bracket-generating if and only if

$$
\mathfrak{g}_{-1} \text { is contained in the algebra generated by } \mathfrak{k}_{-} \oplus \mathfrak{q}_{+} \text {. }
$$

Observe that $\mathfrak{g}_{-1} \subset \mathfrak{g}_{-1}^{\prime}, \mathfrak{g}_{0} \subset \mathfrak{g}_{0}^{\prime}$ (both inclusions usually strict), and

$$
\mathfrak{g}_{-, \text {even }}^{\prime} \subset \mathfrak{g}_{-, \text {even }}=\mathfrak{k}_{-} \quad \text { and } \quad \mathfrak{g}_{+, \text {odd }}^{\prime}=\mathfrak{g}_{+, \text {odd }}=\mathfrak{q}_{+} .
$$

Therefore, by (16), to see that $\breve{S}$ is bracket-generating it suffices to show that

$$
\mathfrak{g}_{-1}^{\prime} \text { is contained in the algebra generated by } \mathfrak{g}_{-, \text {even }}^{\prime} \oplus \mathfrak{g}_{+, \text {odd }}^{\prime} \text {. }
$$

Proof of (17). Note that $\left[\mathfrak{g}_{1}^{\prime}, \mathfrak{g}_{-2}^{\prime}\right]$ is a direct sum of (a subset of) root spaces $\mathfrak{g}^{-\alpha} \subset \mathfrak{g}_{-1}^{\prime}$. Let $\Gamma=\left\{-\alpha \in \Delta\left(\mathfrak{g}_{-1}^{\prime}\right) \mid \mathfrak{g}^{-\alpha} \not \subset\left[\mathfrak{g}_{1}^{\prime}, \mathfrak{g}_{-2}^{\prime}\right]\right\}$, and define

$$
\mathfrak{f}_{-1}=\bigoplus_{-\alpha \in \Gamma} \mathfrak{g}^{-\alpha} \text { and } \mathfrak{f}_{1}=\bigoplus_{-\alpha \in \Gamma} \mathfrak{g}^{\alpha} .
$$

Note that $\mathfrak{g}_{-1}^{\prime}=\left[\mathfrak{g}_{1}^{\prime}, \mathfrak{g}_{-2}^{\prime}\right]$ (and (17) holds) if and only if $\mathfrak{f}_{ \pm 1}=0$. Set $\mathfrak{f}_{0}=\left[\mathfrak{f}_{1}, \mathfrak{f}_{-1}\right]$. Define

$$
\mathfrak{f}=\mathfrak{f}_{1} \oplus \mathfrak{f}_{0} \oplus \mathfrak{f}_{-1} .
$$

We will show that $\mathfrak{f}$ is an ideal of $\mathfrak{g}_{\mathbb{C}}$. Since, by assumption, $\mathfrak{g}_{\mathbb{C}}$ is simple, it follows that either $\mathfrak{f}=0$ or $\mathfrak{f}=\mathfrak{g}_{\mathbb{C}}$. If $\mathfrak{f}=\mathfrak{g}_{\mathbb{C}}$, then $\mathfrak{g}_{ \pm}=\mathfrak{f}_{ \pm 1}$ is abelian. It follows easily that $\mathfrak{f}_{ \pm}=\mathfrak{g}_{ \pm}^{\prime}=\mathfrak{q}_{ \pm}$and $\mathfrak{f}_{0}=\mathfrak{g}_{0}^{\prime}=\mathfrak{k}_{\mathbb{C}}$. Therefore, $G / K$ is Hermitian symmetric, with $(1,0)$-tangent space $\mathfrak{q}_{-}$. Moreover, the derivative of the projection $p: G / V \rightarrow G / K$ is the projection $\mathfrak{k}_{-} \oplus \mathfrak{q}_{-} \rightarrow \mathfrak{q}_{-}$. Thus (recalling (7)) $p$ is holomorphic, contradicting our assumption that $D=G / V$ is non-classical. Therefore, $\mathfrak{f}=0$, and (17) holds, establishing Lemma 2.6. 


\section{Griffiths, Robles And Toledo}

To prove that $\mathfrak{f}$ is an ideal, we proceed in these steps:

Step 1. Note that $\mathfrak{f}_{-1}$ is the maximal subspace of $\mathfrak{g}_{-1}^{\prime}$ with the property that $B\left(\mathfrak{f}_{-1},\left[\mathfrak{g}_{-1}^{\prime}, \mathfrak{g}_{2}^{\prime}\right]\right)=0$. Equivalently,

$$
0=B\left(\mathfrak{f}_{1},\left[\mathfrak{g}_{1}^{\prime}, \mathfrak{g}_{-2}^{\prime}\right]\right)=B\left(\mathfrak{g}_{1}^{\prime},\left[\mathfrak{f}_{1}, \mathfrak{g}_{-2}^{\prime}\right]\right)=B\left(\mathfrak{g}_{-2}^{\prime},\left[\mathfrak{f}_{1}, \mathfrak{g}_{1}^{\prime}\right]\right) .
$$

In particular, $\mathfrak{f}_{1}$ is the largest subspace of $\mathfrak{g}_{1}^{\prime}$ with the property that $\left[\mathfrak{f}_{1}, \mathfrak{g}_{1}^{\prime}\right]=0$; equivalently, $\left[\mathfrak{f}_{-1}, \mathfrak{g}_{2}^{\prime}\right]=0=\left[\mathfrak{f}_{1}, \mathfrak{g}_{-2}^{\prime}\right]$.

Step 2: $\mathfrak{f}$ is a $\mathfrak{g}_{0}^{\prime}$-module. First observe that $\mathfrak{f}_{1}$ is a $\mathfrak{g}_{0}^{\prime}$-module. To see this, let $G_{0}^{\prime}=\{g \in$ $\left.G_{\mathbb{C}} \mid \operatorname{Ad}_{g}\left(\mathfrak{g}_{\ell}^{\prime}\right)=\mathfrak{g}_{\ell}^{\prime}, \forall \ell\right\}$. Then $G_{0}^{\prime}$ is a closed Lie subgroup of $G_{\mathbb{C}}$ with Lie algebra $\mathfrak{g}_{0}^{\prime}$. By Step 1 , $\mathfrak{f}_{1}$ is the largest subspace of $\mathfrak{g}_{1}^{\prime}$ with the property that $\left[\mathfrak{f}_{1}, \mathfrak{g}_{1}^{\prime}\right]=0$. So,

$$
0=\operatorname{Ad}_{G_{0}^{\prime}}\left[\mathfrak{f}_{1}, \mathfrak{g}_{1}^{\prime}\right]=\left[\operatorname{Ad}_{G_{0}^{\prime}} \mathfrak{f}_{1}, \operatorname{Ad}_{G_{0}^{\prime}} \mathfrak{g}_{1}^{\prime}\right]=\left[\operatorname{Ad}_{G_{0}^{\prime}} \mathfrak{f}_{1}, \mathfrak{g}_{1}^{\prime}\right]
$$

implies $\operatorname{Ad}_{G_{0}^{\prime}} \mathfrak{f}_{1}=\mathfrak{f}_{1}$.

An identical argument, with $\mathfrak{f}_{-1}$ in place of $\mathfrak{f}_{1}$, proves that $\mathfrak{f}_{-1}$ is a $\mathfrak{g}_{0}^{\prime}$-module. It follows from $\mathfrak{f}_{0}=\left[\mathfrak{f}_{1}, \mathfrak{f}_{-1}\right]$ and $\operatorname{Ad}_{G_{0}^{\prime}}\left[\mathfrak{f}_{1}, \mathfrak{f}_{-1}\right]=\left[\operatorname{Ad}_{G_{0}^{\prime}} \mathfrak{f}_{1}, \operatorname{Ad}_{G_{0}^{\prime}} \mathfrak{f}_{-1}\right]$ that $\mathfrak{f}$ is a $\mathfrak{g}_{0}^{\prime}-$ module.

Step 3: $\left[\mathfrak{g}_{ \pm 1}^{\prime}, \mathfrak{f}\right] \subset \mathfrak{f}$. To see that $\left[\mathfrak{g}_{1}^{\prime}, \mathfrak{f}\right] \subset \mathfrak{f}$, we consider each component of $\mathfrak{f}=\mathfrak{f}_{1} \oplus \mathfrak{f}_{0} \oplus \mathfrak{f}_{-1}$ :

- By Step $1,\left[\mathfrak{g}_{1}^{\prime}, \mathfrak{f}_{1}\right]=0$.

- To see that $\left[\mathfrak{g}_{1}^{\prime}, \mathfrak{f}_{-1}\right] \subset \mathfrak{f}_{0}$, let $\mathfrak{f}_{0}^{\perp}$ be the Killing orthogonal complement to $\mathfrak{f}_{0}$ in $\mathfrak{g}_{0}^{\prime}$. Then

$$
0=B\left(\mathfrak{f}_{0}, \mathfrak{f}_{0}^{\perp}\right)=B\left(\left[\mathfrak{f}_{1}, \mathfrak{f}_{-1}\right], \mathfrak{f}_{0}^{\perp}\right)=B\left(\mathfrak{f}_{1},\left[\mathfrak{f}_{-1}, \mathfrak{f}_{0}^{\perp}\right]\right) .
$$

By Step $2, \mathfrak{f}_{-1}$ is a $\mathfrak{g}_{0}^{\prime}$-module. Since $\mathfrak{f}_{0}^{\perp} \subset \mathfrak{g}_{0}^{\prime}$, we must have $\left[\mathfrak{f}_{-1}, \mathfrak{f}_{0}^{\perp}\right] \subset \mathfrak{f}_{-1}$. Then $(*)$ yields $\left[\mathfrak{f}_{-1}, \mathfrak{f}_{0}^{\perp}\right]=0$. It follows that

$$
B\left(\left[\mathfrak{g}_{1}^{\prime}, \mathfrak{f}_{-1}\right], \mathfrak{f}_{0}^{\perp}\right)=B\left(\mathfrak{g}_{1}^{\prime},\left[\mathfrak{f}_{-1}, \mathfrak{f}_{0}^{\perp}\right]\right)=0,
$$

yielding $\left[\mathfrak{g}_{1}^{\prime}, \mathfrak{f}_{-1}\right] \subset \mathfrak{f}_{0}$.

- The Jacobi identity implies that $\left[\mathfrak{g}_{1}^{\prime}, \mathfrak{f}_{0}\right]=\left[\mathfrak{g}_{1}^{\prime},\left[\mathfrak{f}_{1}, \mathfrak{f}_{-1}\right]\right] \subset \mathfrak{f}$.

We conclude that $\left[\mathfrak{g}_{1}^{\prime}, \mathfrak{f}\right] \subset \mathfrak{f}$. A similar argument yields $\left[\mathfrak{g}_{-1}^{\prime}, \mathfrak{f}\right] \subset \mathfrak{f}$, completing Step 3 .

Step 4: Induction. Suppose that $\left[\mathfrak{g}_{\ell}^{\prime}, \mathfrak{f}\right] \subset \mathfrak{f}$ for some $\ell>0$. Since $\mathfrak{g}_{1}^{\prime}$ generates $\mathfrak{g}_{+}^{\prime}$, we have $\mathfrak{g}_{\ell+1}^{\prime}=\left[\mathfrak{g}_{\ell}^{\prime}, \mathfrak{g}_{1}^{\prime}\right]$. Then the Jacobi identity yields $\left[\mathfrak{g}_{\ell+1}^{\prime}, \mathfrak{f}\right] \subset \mathfrak{f}$. Thus $\left[\mathfrak{g}_{+}^{\prime}, \mathfrak{f}\right] \subset \mathfrak{f}$. A similar argument yields $\left[\mathfrak{g}_{-}^{\prime}, \mathfrak{f}\right] \subset \mathfrak{f}$.

Fini. This completes the proof that $\mathfrak{f}$ is an ideal of $\mathfrak{g}_{\mathbb{C}}$, and establishes (17), and thus Lemma 2.6.

\section{Proof of Theorem 1.2}

Let $E, F, S$ be as in Definition 2.3, and let $\mathcal{E}, \mathcal{F}, \mathcal{S}$ be their respective spaces of smooth sections. We are interested in the collection $\mathcal{E} \cup \mathcal{F}$ of vector fields on $\mathcal{I}$ and in the pseudogroup $\mathcal{G}$ generated by their flows:

Definition 4.1. Let $\mathcal{G}$ be the collection of all diffeomorphisms of $\mathcal{I}$ of the form

$$
\Phi(\vec{X}, \vec{t})=\exp \left(t_{k} X_{k}\right) \circ \ldots \circ \exp \left(t_{2} X_{2}\right) \circ \exp \left(t_{1} X_{1}\right)
$$

where $\vec{X}=\left(X_{1}, \ldots X_{k}\right)$, each $X_{i} \in \mathcal{E} \cup \mathcal{F} ; \vec{t}=\left(t_{1}, \ldots t_{k}\right)$, each $t_{i} \in \mathbb{R}$, and $k$ is a natural number.

Given $(x, u) \in \mathcal{I}$, by the orbit of $(x, u)$ under $\mathcal{G}$, we mean the set $\mathcal{G}(x, u)=\{\Phi(\vec{X}, \vec{t})(x, u)$ : all $\vec{X}, \vec{t}$ as above $\}$. 


\section{NON-CLASSICAL DOMAINS}

We have the following proposition:

Proposition 4.2. For any $\left(x_{0}, u_{0}\right) \in \mathcal{I}$, the orbit $\mathcal{G}\left(x_{0}, u_{0}\right)=\mathcal{I}$.

Proof. Let $\mathcal{L}(\mathcal{E} \cup \mathcal{F})$ denote the Lie subalgebra of the algebra $C^{\infty}(T \mathcal{I})$ of smooth vector fields on $\mathcal{I}$ generated by $\mathcal{E} \cup \mathcal{F}$. For each $(x, u) \in \mathcal{I}$, let $\mathcal{L}_{(x, u)}(\mathcal{E} \cup \mathcal{F}) \subset T_{(x, u)} \mathcal{I}$ denote the set of evaluations at $(x, u)$ of all the vector fields in $\mathcal{L}(\mathcal{E} \cup \mathcal{F})$. A standard theorem in Control Theory, going back to [St74, Su73], asserts:

If $\mathcal{L}(\mathcal{E} \cup \mathcal{F})=\mathcal{T}_{(x, u)} \mathcal{I}$, for all $(x, u) \in \mathcal{I}$, then $\mathcal{G}$ has only one orbit; namely $\mathcal{I}$.

(Cf. Theorem 3 of [Jur97, Section 2.3].) So to prove the proposition, it suffices to show the following:

$$
\text { For all }(x, u) \in \mathcal{I} \text {, we have } \mathcal{L}_{(x, u)}(\mathcal{E} \cup \mathcal{F})=T_{(x, u)} \mathcal{I} \text {. }
$$

By Lemma 2.5, $E \oplus F=S$. So, the Lie algebra generated by $\mathcal{E} \cup \mathcal{F}$ is the same as the Lie algebra $\mathcal{L}(\mathcal{S})$ generated by $\mathcal{S}$. By Lemma 2.6, $S$ is bracket-generating. Thus, for all $(x, u) \in \mathcal{I}$, $\mathcal{L}_{(x, u)}(\mathcal{S})=T_{(x, u)} \mathcal{I}$; this establishes $(18)$.

Now we can complete the proof of Theorem 1.2. Let $\left(x_{0}, u_{0}\right) \in \mathcal{I}$, let $\Phi(\vec{X}, \vec{t}) \in \mathcal{G}$ be as in Definition 4.1, and, for each $j=1, \ldots, k$, let

$$
\left(x_{j}, u_{j}\right)=\exp \left(t_{j} X_{j}\right) \circ \ldots \circ \exp \left(t_{1} X_{1}\right)\left(x_{0}, u_{0}\right)
$$

so that

$$
\left(x_{j+1}, u_{j+1}\right)=\exp \left(t_{j+1} X_{j+1}\right)\left(x_{j}, u_{j}\right) .
$$

Note that each step (20) is obtained by flowing along the vector field $X_{j+1}$ : let $\gamma(t)$ be the curve $\exp \left(t X_{j+1}\right)\left(x_{j}, u_{j}\right)$. Then $\gamma(0)=\left(x_{j}, u_{j}\right)$ and $\gamma\left(t_{j+1}\right)=\left(x_{j+1}, u_{j+1}\right)$. We will describe each step (20) by saying $\left(x_{j}, u_{j}\right)$ flows along $X_{j+1}$ to $\left(x_{j+1}, u_{j+1}\right)$.

Since $X_{j+1} \in \mathcal{E} \cup \mathcal{F}$, in each step (20) either $u$ is constant or $x$ is constant. That is, either:

- $u_{j}=u_{j+1}$ and $x$ flows from $x_{j}$ to $x_{j+1}$ inside $Z_{u_{j}}$, if $X_{j+1} \in \mathcal{E}$.

- $x_{j}=x_{j+1}$ and $Z_{u}$ flows from $Z_{u_{j}}$ to $Z_{u_{j+1}}$ keeping $x_{j}$ fixed, if $X_{j+1} \in \mathcal{F}$.

Therefore the points $x_{0}$ and $x_{k}$ are joined by a chain of subvarieties $Z_{u_{0}}, \ldots, Z_{u_{k}}$ where each subvariety meets the next. (Maybe one is identical to the next, in which case we may want to eliminate repetitions). Since $x_{0}, x_{k} \in D$ are arbitrary, Theorem 1.2 is proved.

\section{Proof of Theorem 1.1}

Let $D$ be a non-classical domain, let $\Gamma \subset G$ be an infinite, finitely generated discrete subgroup. Then $X=\Gamma \backslash D$ is a normal analytic space, and we want to prove that $X$ does not have an algebraic structure.

\subsection{Reduction to torsion-free $\Gamma$}

Since $\Gamma$ is a finitely generated linear group, it has a torsion-free subgroup $\Gamma^{\prime}$ of finite index (Proposition 2.3 of [Bor63]). Then $\Gamma^{\prime}$ acts freely on $D=G / V$ and the quotient space $X^{\prime}=\Gamma^{\prime} \backslash D$ is a complex manifold with fundamental group $\Gamma^{\prime}$. The natural projection $\pi: X^{\prime} \rightarrow X$ is a finite analytic map, that is, a proper analytic map with finite fibers.

Lemma 5.1. Let $\gamma \in G$ be an element of finite order, $\gamma \neq e$. Then the fixed point set of $\gamma$ is a complex submanifold of $D$ of complex codimension at least two. 


\section{Griffiths, Robles And Toledo}

Proof. If $\gamma$ has a fixed point in $G / V$ then it is conjugate in $G$ to an element of $V$, so we may assume $\gamma \in V$. If a group $H$ acts on a space $A$ and $h \in H$, let $F(h, A)$ denote the fixed point set of $h$ in $A$. Since $\gamma$ is a holomorphic map of $D$ and is an isometry of a Hermitian metric ( $\S 9$ of [GS69]), $F(\gamma, D)$ is a complex submanifold of $D$. We claim that $F(\gamma, D)$ is fibered as follows by the restriction of the projection $p: D=G / V \rightarrow G / K$ :

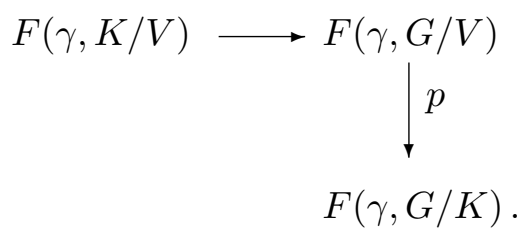

This is easily checked by writing explicitly $p \mid F(\gamma, D)$ :

$$
\left\{g V \in G / V: g^{-1} \gamma g \in V\right\} \rightarrow\left\{g K \in G / K: g^{-1} \gamma g \in K\right\}
$$

which takes $g V$ to $g K$. This map is surjective because the fiber over $g K \in F(\gamma, G / K)$ is

$$
\left\{g k V: k \in K \text { and } k^{-1} g^{-1} \gamma g k \in V\right\}=g F\left(g^{-1} \gamma g, K / V\right),
$$

which is not empty, because $K$ is compact and $T \subset V$, so $g^{-1} \gamma g$ is conjugate to an element of $T$. Thus $\left.p\right|_{F(\gamma, G / V)}$ is a fibration of $F(\gamma, D)$ over $F(\gamma, G / K)$ with fibers biholomorphic to $F(\gamma, K / V)$.

Recall we are assuming that $G$ is a simple adjoint group. Since it has no non-trivial normal subgroups, it must act effectively on $D=G / V$ and on the symmetric space $G / K$. But $K$ must have non-trivial center since it contains the Cartan involution. Recall that $K / V$ is always a positive-dimensional complex manifold, since $D$ is non-classical. In particular, $K$ is non-abelian.

There are two cases to consider:

Case 1: $\gamma$ does not fix $K / V$ pointwise. Then the real codimension of $F(\gamma, K / V)$ in $K / V$ is at least two, since it is a proper complex submanifold of the positive-dimensional complex manifold $K / V$. The real codimension of $F(\gamma, G / K)$ is at least one, since it is a proper submanifold of $G / K$. Therefore the real codimension of $F(\gamma, D)$ is at least three, so its complex codimension is at least two.

Case 2: $\gamma$ fixes $K / V$ pointwise. Then $\gamma \in N=\cap_{k \in K} k V k^{-1}$, which is a non-trivial proper normal subgroup of $K$ containing the center $Z(K)=\cap_{k \in K} k T k^{-1}$ of $K$, that is $Z(K) \subset N \subsetneq V \subsetneq K$.

Recall that $K$ is connected and is either a simple group, or finitely covered by a product of compact simple groups $K_{1}, K_{2}, \ldots$ or, exactly when $G / K$ is Hermitian symmetric, it is finitely covered by a product $S^{1} \times K^{\prime}$ where $K^{\prime}$ is a product of simple groups and the image of $S^{1}$ is the center of $K$.

We claim that $Z_{K}(\gamma)$, the centralizer of $\gamma$ in $K$, always contains a non-abelian subgroup $L$ of $K$ which is a product of simple factos of $K$. To prove this, lift to a finite cover $\hat{K}=Z K_{1} \ldots K_{m}$ which is a product, with $Z$ central and the $K_{i}$ compact simple. Then the proper normal Lie subgroup $\hat{N}$ with connected component $\hat{N}^{0}$ is contained in the product of $\hat{N}^{0}$ with the centers of the remaining factors. These factors centralize $\hat{N}$, hence $\gamma$. Call their product $L$. Then $L$ leaves $F(\gamma, G / K)$ invariant. Then the tangent space $T_{e K} F(\gamma, G / K)$ is a proper $L$-invariant subspace of $T_{e K} G / K$. It has a non-zero $L$-invariant complement. Since $G / K$ is irreducible, the action of $K$ on the tangent space $T_{e K} G / K$ is irreducible. This implies that the factor $L$ of $K$ cannot act trivially on any non-zero subspace of $T_{e K} G / K$. (If it did, the subspace of $L$-invariant vectors in $T_{e K} G / K$ would be a non-zero, proper subspace stable under $K$, contradicting irreducibility.) 


\section{NON-CLASSICAL DOMAINS}

Therefore the $L$-invariant complement of $T_{e K} F(\gamma, G / K)$ is a non-trivial representation of the real semisimple group $L$, hence of dimension at least three, so the real codimension of $F(\gamma, G / K)$ in $G / K$ is at least three. Therefore the real codimension of $F(\gamma, G / V)$ must be at least three, so its complex codimension at least two.

Applying this lemma to the elements of finite order in $\Gamma$, we see that there are analytic subsets $Y, Y^{\prime}$ of codimension at least two, $Y^{\prime} \subset X^{\prime}$ and $Y \subset X$, so that $\pi: X^{\prime} \backslash Y^{\prime} \rightarrow X \backslash Y$ is unramified. Moreover $Y$ is the singular set of the normal analytic space $X$.

Suppose $X$ has an algebraic structure, meaning that there is an algebraic variety $W$ so that $X=W^{\text {an }}$ is the analytic space associated to $W$. Then $Y=\Sigma^{\text {an }}$, where $\Sigma \subset W$ is the singular set of $W$. Let $U=W \backslash \Sigma$ be the set of regular points of $W$. Since $\left.\pi\right|_{X^{\prime} \backslash Y^{\prime}}$ is finite étale, by the "Riemann Existence Theorem" (Theorem XII 5.1 of [Gro71]), $X^{\prime} \backslash Y^{\prime}$ would also have an algebraic structure. In other words, there would be an algebraic variety $U^{\prime}$ so that $U^{\prime \text { an }}=X^{\prime} \backslash Y^{\prime}$ and a finite étale map $\rho: U^{\prime} \rightarrow U$ with $\rho^{\text {an }}=\pi$.

Let $j: U \rightarrow W$ be the inclusion. Since $\Sigma$ has codimension at least two, $j_{*} \rho_{*} \mathcal{O}_{U^{\prime}}$ is a coherent sheaf of algebras over $\mathcal{O}_{W}$ whose associated analytic sheaf on $X=W^{\text {an }}$ agrees with $\pi_{*} \mathcal{O}_{X^{\prime}}$ on $X \backslash Y$, hence on all of $X$. Then $W^{\prime}=\operatorname{Spec}_{W}\left(j_{*} \rho_{*} \mathcal{O}_{U^{\prime}}\right)$ is a scheme of finite type over $\mathbb{C}$ with $W^{\prime \text { an }}=X^{\prime}$.

In other words, if $X$ were algebraic, $X^{\prime}$ would also be algebraic. So we may assume that our discrete group $\Gamma$ is torsion-free.

\subsection{The Shafarevich Map}

Assume from now on that $X=\Gamma \backslash D$ where $\Gamma$ has no torsion, thus $X$ is a complex manifold with fundamental group $\Gamma$. Suppose that $X$ is an algebraic variety. We will derive a contradiction by using Kollár's Shafarevich map. We begin by recalling the facts that we need from [Ko95].

First, according to Definition 3.5 of [Ko95], (specialized to the case $H=\{$ id $\}$ ), if $X$ is a normal algebraic variety, a Shafarevich variety $S h(X)$ and Shafarevich map $s h_{X}$ are a normal variety $S h(X)$ and a rational map $s h_{X}: X \rightarrow S h(X)$ with the following properties: $s h_{X}$ has connected fibers and there is a countable collection of closed subvarieties of $D_{i} \subset X, D_{i} \neq X$, so that for any closed, irreducible subvariety $Z \subset X$ not contained in $\cup D_{i}$ the following holds:

$$
s h_{X}(Z)=\text { point if and only if } \operatorname{im}\left\{\pi_{1}(\bar{Z}) \rightarrow \pi_{1}(X)\right\} \text { is finite, }
$$

where $\bar{Z}$ denotes the normalization of $Z$.

Definition 5.2 (Definition 2.1 of [Ko95]). A normal cycle in $X$ means an irreducible normal variety $W$ together with a finite morphism $w: W \rightarrow X$ that is birational to its image.

Theorem 5.3 (Theorem 3.6 of [Ko95]). Let $X$ be a normal algebraic variety. Then a Shafarevich variety and map exist, unique up to birational equivalence. Moreover, for every choice of $\operatorname{Sh}(X)$ within its birational equivalence class, there are Zariski open subsets $X_{0} \subset X$ and $Y^{0} \subset S h(X)$ so that

(a) $s h_{X}: X^{0} \rightarrow Y^{0}$ is everywhere defined,

(b) Every fiber of $s h_{X} \mid X^{0}$ is closed in $X$,

(c) $s h_{X} \mid X^{0}$ is a topologically locally trivial fibration.

(d) Let $y \in Y^{0}$ be very general, let $X_{y}^{0}$ be the fiber of $\operatorname{sh}_{X} \mid X^{0}$ over $y$, and let $w: W \rightarrow X$ be a normal cycle with $\operatorname{im}\left\{\pi_{1}(W) \rightarrow \pi_{1}(X)\right\}$ finite. If $\operatorname{im}\{w\} \cap X_{y}^{0} \neq \emptyset$, then $\operatorname{im}\{w\} \subset X_{y}^{0}$. 


\section{Griffiths, Robles And Toledo}

Remark 5.4. The fourth statement does not appear explicitly in the statement of Theorem 3.6 of [Ko95], but it appears in Corollary 3.4 and in the proof of Theorem 3.6, and is used in the proof of Theorem 4.13. It is a more detailed version of (21). (We have also omitted statements regarding proper varieties, since our interest is in the non-proper situation.)

Proof of Theorem 1.1. We now suppose that the complex manifold $X=\Gamma \backslash D$ has a compatible algebraic structure and derive a contradiction by using Theorem 5.3. We first prove that $s h_{X} \mid X^{0}$ is constant.

Let $\pi: D \rightarrow X$ be the projection. For any of our compact subvarieties $Z_{u} \subset D$, the map $\pi \mid Z_{u}: Z_{u} \rightarrow X$ is an immersion, therefore it is finite and birational to its image, in other words, it is a normal cycle in the sense of Definition 5.2.

Let $y_{1}, y_{2} \in Y^{0}$ be very general points. Choose $x_{1}, x_{2} \in D$ so that $\pi\left(x_{1}\right) \in X_{y_{1}}^{0}$ and $\pi\left(x_{2}\right) \in$ $X_{y_{2}}^{0}$. By Theorem 1.2 there is a chain $u_{1}, \ldots, u_{k} \in \mathcal{U}$ so that, letting $Z_{i}$ denote $Z_{u_{i}}, x_{1} \in$ $Z_{1}, x_{2} \in Z_{k}$ and $Z_{i} \cap Z_{i+1} \neq \emptyset$. Choose a point $z_{i} \in Z_{i} \cap Z_{i+1}$. Let $w_{i}$ denote the normal cycle $w_{i}=\pi \mid Z_{i}: Z_{i} \rightarrow X$. Recall that $Z_{i}=K / V$ is simply connected. By (d) of Theorem 5.3, $\operatorname{im}\left\{w_{1}\right\} \subset X_{y_{1}}^{0}$, hence $w_{1}\left(z_{1}\right) \in X_{y_{1}}^{0}$, hence $\operatorname{im}\left\{w_{2}\right\} \subset X_{y_{1}}^{0}$. Continuing this way, we get $w_{k}\left(x_{2}\right) \in X_{y_{1}}^{0}$. But by definition $w_{k}\left(x_{2}\right) \in X_{y_{2}}^{0}$. Hence $y_{1}=y_{2}$ for any two very general points in $Y^{0}$. Thus $s h_{X} \mid X^{0}: X^{0} \rightarrow Y^{0}$ is the constant map.

Now, by Theorem 5.3(b), the single fiber $X^{0}$ of $s h_{X}$ is closed in $X$, hence $X^{0}=X$, the rational map $s h_{X}$ is everywhere defined on $X$ and $s h_{X}(X)$ is a point. Therefore (21), gives that $\pi_{1}(X)$ is finite. But $\pi_{1}(X) \cong \Gamma$ is infinite, thus contradicting the algebraicity of $X$.

\section{ACKNOWLEDGEMENTS}

We thank János Kollár for pointing us to Theorem 3.6 of [Ko95] and for several very helpful conversations, comments and concrete suggestions. We thank Igor Zelenko for pointing us to [Jur97] and the referee for pointing out that Theorem 1.2 had already been proved by Huckleberry in [Hu13], and for other helpful comments.

\section{REFERENCES}

Bor63 A. Borel, Compact Clifford-Klein forms of symmetric spaces, Topology 2 (1963), 111-122.

Cam91 F. Campana, On twistor spaces of class $\mathcal{C}$, J. Differential Geom. 33 (1991), 541-549.

CT12 J.A. Carlson and D. Toledo, Compact quotients of non-classical domains are not Kähler, to appear in the proceedings of the CBMS Conference on Hodge Theory, Complex Geometry and Representation Theory, Texas Christian University, June 2012.

CS09 A. Čap and J. Slovák, Parabolic Geometries, I, Mathematical Surveys and Monographs, 154, American Mathematical Society, Providence, RI, 2009.

FHW06 G. Fels, A. Huckleberry and J.A. Wolf, Cycle Spaces of Flag Domains, Progress in Mathematics, 245, Birkhäuser, 2006.

Gr12 P.A. Griffiths, Hodge Theory and Representation Theory, Notes for the CBMS Conference on Hodge Theory, Complex Geometry and Representation Theory at Texas Christian University, June 2012.

GS69 P.A. Griffiths and W. Schmid, Locally homogeneous complex manifolds, Acta Math. 123 (1969), 253-302.

Gro71 A. Grothendieck, Revêtements Étales et Groupe Fondamental, SGA 1, Lecture Notes in Mathematics 224, Springer Verlag, 1971. 


\section{NON-CLASSICAL DOMAINS}

Hu13 A. Huckleberry, Hyperbolicity of cycle spaces and automorphism groups of flag domains, Amer. J. Math. 135 (2013), 291-310. http://dx.doi.org/10.1353/ajm.2013.0016

Jur97 V. Jurdjevic, Geometric Control Theory, Cambridge Studies in Advanced Mathematics, Cambridge University Press, 1997.

Ko95 J. Kollár, Shafarevich Maps and Automorphic Forms, Princeton University Press, 1995.

St74 P. Stefan, Accessible sets, orbits and foliations with singularities, Proc. London Math. Soc. 29 (1974), 699-713.

Su73 H. Sussmann, Orbits of families of vector fields and integrability of distributions, Trans. Amer. Math. Soc. 180 (1973), 171-188.

WW77 R.O. Wells and J.A. Wolf, Poincaré series and automorphic cohomology on flag domains, Ann. of Math. 105 (1977), 397-448.

Wo69 J.A. Wolf, The action of a real semisimple group on a complex flag manifold, I: Orbit structure and holomorphic arc components, Bull. Amer. Math. Soc. 75 (1969), 1121-1237.

Phillip Griffiths pg@ias.edu

School of Mathematics, Institute for Advanced Study, Einstein Drive, Princeton, NY 08540, USA

Colleen Robles robles@math.tamu.edu

Mathematics Department, Mail-stop 3368, Texas A\&M University, College Station, TX 778433368, USA

Domingo Toledo toledo@math.utah.edu

Mathematics Department, University of Utah, Salt Lake City, Utah 84112, USA 